\title{
EDITORIAL
}

\section{Estimating prognosis in heart failure: time for a better approach}

\section{R Cowie}

Heart 2003;89:587-588

More research is needed into estimating risk in heart failure and in communicating prognostic information to patients

\footnotetext{
E
} stimating prognosis is endorsed by recent clinical guidelines as a key element of the management of heart failure. ${ }^{12}$ How this is to be done is less clear. Heart failure follows a variable clinical course, and as many as one half of patients will die suddenly rather than dying of progressive heart failure. ${ }^{3}$ The predictability of death may therefore be lower than in other conditions. The challenge of identifying clinical variables consistently associated with mortality has been summarised by Cowburn and colleagues. ${ }^{4}$ Many of the published studies are small, and include highly selected patient populations, with different variables recorded in each dataset. Differences in the way measurements are made-for example, in the assessment of left ventricular systolic function-and the close correlation between many variables add further complexity. Also, it should not be assumed that the same variables will be associated with mortality in the early phases of heart failure as well as in the later phases. The recent rise in the use of $\beta$ blockade may make the conclusions drawn from earlier studies less relevant to modern practice. From the statistical viewpoint, the methodology of how best to use available data and to validate the derived risk equations is rapidly evolving. ${ }^{56}$

Writing in this issue of Heart, Bouvy and colleagues are to be congratulated on recognising the need for a simple, but robust, prognostic scoring system for patients with heart failure. ${ }^{7}$ Simple models are not necessarily of less value than more complex models. ${ }^{8}$ Indeed, they may be closer to real life practice. Many patients with heart failure are not exposed to "high tech" investigations. For example, the Euroheart heart failure survey reported that less than $5 \%$ of patients with heart failure who come into contact with a hospital undergo a cardiopulmonary exercise test. Although maximal oxygen consumption and other measures of exercise performance may be useful predictors of survival such data are unlikely to be available for most patients.

Correspondence to: Professor Martin R Cowie, Cardiac Medicine, National Heart \& Lung Institute, London SW3 6LY, UK; m.cowie@ imperial.ac.uk

\section{A CLINICAL APPROACH}

In day-to-day practice the process of arriving at a diagnosis is hierarchical. The physician proceeds by gathering information from history, then physical examination, supplemented by increasingly complex tests before reaching a point where the diagnosis in question is considered either very likely or very unlikely. Bouvy and colleagues adopt a similar approach to estimating the probability of survival. ${ }^{7}$ The initial statistical model uses only information from the clinical history. They then add features from physical examination and the results of simple laboratory investigations. Only where there is good evidence that more sophisticated tests or less readily available information add to the prediction of risk do they include these. In addition, they recognise that dropping patients with missing data introduces bias and reduces the power of their model. Instead they impute values for those that are missing, an increasingly accepted approach that if done properly can be of great value. ${ }^{5}$

The Bouvy score is practical and potentially useful in a wide range of clinical and health care settings. The information required to derive the score is age, sex, New York Heart Association (NYHA) class, history of diabetes mellitus, history of renal dysfunction, weight, blood pressure, ankle oedema, and use of $\beta$ blockers. Statistical tests for "goodness-of-fit" of the model (how well the predicted mortality in the model accords with the observed mortality) appear satisfactory. The ability of the score to discriminate between those who died and those who survived during 18 months of follow up is very good (area under the receiver operating characteristics curve is 0.84 , $95 \%$ confidence interval 0.77 to 0.90 ). This compares favourably with many diagnostic and prognostic scoring systems generally accepted in other disease areas. ${ }^{9}$

\section{WILL THE SCORE WORK IN PRACTICE?}

A scoring system is only of clinical value if it works when transferred into a population other than the one from which it was derived. Bouvy and colleagues recruited a cohort of patients similar to those reported from population based studies $^{10}$ - the average age was 70 years, two thirds were female, and the single most common aetiology was coronary artery disease. Of note, a third of their patients were treated with a $\beta$ blocker-a much higher proportion than in earlier published reports of prognostic variables. ${ }^{4}$ However, the patients were recruited from a randomised clinical trial and may therefore not be truly representative of patients with heart failure. The results look promising, particularly as the component variables have been reported to be associated with survival in other studies. ${ }^{41}$ External validation in another dataset is required and is underway. 


\section{THE FUTURE FOR RISK PREDICTION}

Is it valuable to spend more research effort on developing better risk prediction equations for patients with heart failure? The evolving professional and societal consensus is that doctors should be more open with patients about all possible courses of action and the likely results of these actions. If a doctor does not communicate prognostic information to a patient they may be denying the patient an opportunity to make fully informed choices about their care. Inaccurate estimates of prognosis may distort a patient's choice of treatment and the trade-off that they may be willing to make between quality and quantity of life-a particularly important consideration for very elderly patients with heart failure. Most patients do not need a precise understanding of their prognosis to change their treatment preferences-studies involving patients with cancer suggest that merely telling a patient that they have at least a $10 \%$ probability of not surviving six months may make them substantially alter their treatment preferences. ${ }^{12}$ Recent studies report that most people with heart failure do not have a discussion with health care professionals about the prognosis of their disease. ${ }^{13}{ }^{14}$ We owe it to our patients to develop tools that enable us to predict prognosis as precisely and as accurately as is biologically possiblerecognising that this will always be an inexact science. The communication of this information, and helping patients to arrive at a truly informed choice about their care, should be an integral part of clinical management.

\section{A CHALLENGE FOR GUIDELINES AND PROFESSIONALS}

At a more pragmatic level, doctors and other health care professionals need clear guidance on how to identify patients at particularly high or low risk. This should then trigger changes in care, with consideration of referral for appropriately aggressive or intensive therapy. Such treatment may best be delivered in another health care setting or by another health care professional. Clinical guidelines need to address these practical issues if they are to impact properly on the care of patients with heart failure. Estimating prognosis, talking with patients about these issues, and arriving at an appropriate management plan is a challenge. The work of Bouvy and colleagues $^{7}$ is a welcome step towards a more objective and rational approach.

\section{REFERENCES}

1 Task Force for the Diagnosis and Treatment of Chronic Heart Failure ESC. Guidelines for the diagnosis and treatment of chronic heart failure. Eur Heart J 2001;22:1527-60.

2 American College of Cardiology, American Heart Association. $\mathrm{ACC} / \mathrm{AHA}$ guidelines for the evaluation and management of chronic heart failure in the adult: a report of the American College of Cardiology/American Heart Association task force on practice guidelines (committee to revise the 1995 guidelines for the evaluation and management of heart failure). Accessed 23 October 2002. URL: http://www.acc.org/clinical/guidelines/failure/hf_index.htm

3 Cleland JGF, Massie BM, Packer, M. Sudden death in heart failure: vascular or electrical. Eur J Heart Failure 1999;1,41-5.

4 Cowburn PJ, Cleland JGF, Coats AJS, et al. Risk stratification in chronic heart failure. Eur Heart J 1998;19:696-710.

5 Harrell FE, Lee KL, Mark DB. Tutorials in biostatistics - multivariable prognostic models: issues in developing models, evaluating assumptions and adequacy, and measuring and reducing errors. Statist Med 1996; 15:361-87.

6 Greenland S, Finkle WD. A critical look at methods for handling missing covariates in epidemiologic regression analyses. Am J Epidemiol 1995; 142: 1255-64.

7 Bouvy ML, Heerdink ER, Leufkens HGM, et al. Predicting mortality in patients with heart failure: a pragmatic approach. Heart 2003;89:605-9.

8 Zugck C, Kruger C, Kell R, et al. Risk stratification in middle-aged patients with congestive heart failure: prospective comparison of the heart failure survival score (HFSS) and a simplified two variable model. Eur J Heart Failure $2001 ; 3: 577-85$.

9 Mackillop WJ, Quirt CF. Measuring the accuracy of prognostic judgements in oncology. J Clin Epidemiol 1997;50:21-9.

10 Cowie MR, Mosterd A, Wood DA, et al. The epidemiology of heart failure. Eur Heart J 1997; 18:208-15.
11 Cowie MR, Wood DA, Coats A, et al. Survival of patients with a new diagnosis of heart failure: a population-based study. Heart 2000;83:505-10.

12 Weeks JC, Cook EF, O'Day SJ, et al. Relationship between cancer patients' predictions of prognosis and their treatment preferences. JAMA 1998;279: 1709-14.

13 Murray SA, Boyd K, Kendall M, et al. Dying of lung cancer or cardiac failure: prospective qualitative interview study of patients and their carers in the community. BMV 2002;325:929-39.

14 Rogers AE, Addington-Hall J, Abery AJ, et al. Knowledge and communication difficulties for patients with chronic heart falure: qualitative study. BN 2000;321:605-7.

\section{WEB TOP 10}

\section{www.heartinl.com}

These articles scored the most hits on Heart's website during March 2003

1 Management of ischaemic mitral regurgitation $B$ lung

April 2003;89:459-64. (Education in Heart)

2 Position and Mobilisation Post-Angiography Study (PAMPAS): a comparison of 4.5 hours and 2.5 hours bed rest

SD Pollard, K Munks, C Wales, DC Crossman,

DC Cumberland, GDG Oakley, J Gunn

April 2003;89:447-8. (Scientific letter)

3 Differential diagnosis of cardiogenic syncope and seizure disorders

L Bergfeldt

March 2003;89:353-8. (Education in Heart)

4 Lipid lowering to delay the progression of coronary artery disease

MD Feher

April 2003;89:451-8. (Education in Heart)

5 Diagnosis of culture negative endocarditis: novel strategies to prove the suspect guilty

CK Naber, $R$ Erbel

March 2003;89:241-3. (Editorial)

6 Challenges in the care of adult patients with congenital heart defects

GD Webb

April 2003;89:465-9. (Education in Heart)

7 Multicentre evaluation of the diagnostic value of cardiac troponin T, CK-MB mass, and myoglobin for assessing patients with suspected acute coronary syndromes in routine clinical practice

PO Collinson, PJ Stubbs, A-C Kessler

March 2003;89:280-6. (Cardiovascular medicine)

8 Contrast echocardiography

M Stewart

March 2003;89:342-8. (Education in Heart)

9 Evolution of statin prescribing 1994-2001: a case of agism but not of sexism?

S DeWilde, IM Carey, SA Bremner, N Richards,

SR Hilton, D G Cook

April 2003;89:417-21. (Cardiovascular medicine)

10 Clinical assessment of left ventricular diastolic

function

DG Gibson, DP Francis

February 2003;89:231-8. (Education in Heart)

Visit the Heart website for hyperlinks to these articles, by clicking on "Top 10 papers"

www.heartinl.com 